\title{
Absetzerscheinungen bei der Unterbrechung einer SSRI-Behandlung
}

Fragestellung: Welche Rolle spielen Absetzerscheinungen bei Unterbrechungen in der Behandlung mit selektiven SerotoninWiederaufnahmehemmern (SSRI) und können Aussagen in Bezug auf Einsetzen/Dauer der Symptomatik sowie möglicher qualitativer und quantitativer Unterschiede zwischen den einzelnen Wirkstoffen innerhalb der Wirkstoffklasse getroffen werden?

Hintergrund: SSRI sind in der klinischen Praxis häufig verwendete Arzneimittel. Die Einnahme ist mit einem breiten Spektrum an physischen und psychischen Symptomen assoziiert, deren klinische Bedeutung bislang noch nicht vollständig verstanden ist. Bereits 1998 wurde zur Erfassung von Absetzerscheinungen eine spezifische Checkliste (DESS) entwickelt. Diagnostische Kriterien unter Berücksichtigung somatischer und psychischer Symptome wurden von Black

Fava GA, Gatti A, Belaise C et al. Withdrawal symptoms after selective serotonin reuptake inhibitor discontinuation: A systematic review. Psychother Psychosom 2015; 84: 72 - 81 et al. vorgeschlagen. Trotz der verbreiteten Anwendung gab es bislang kein systematisches Review, das sich mit den klinischen Aspekten einer diskontinuierlichen Gabe von SSRI befasst.
Patienten und Methodik: Die Erstellung des systematischen Reviews erfolgte unter Berücksichtigung der PRISMA-Guidelines. Die Suche nach geeigneten Titeln, Abstracts und Themen erfolgte nach vordefinierten Suchbegriffen in den Datenbanken CINAHL, Cochrane Library, PubMed und Web of Science bis Juli 2014. Es wurden nur englischsprachige Studien einbezogen.

Ergebnisse: Die Literaturrecherche ergab 15 geeignete RCT, vier offene Studien, vier retrospektive Studien sowie 38 klinische Fallberichte, die sich unmittelbar und mittelbar mit dem Vorliegen von Absetzerscheinungen bei Unterbrechung der Behandlung mit SSRI befassten. Die Prävalenz der Absetzerscheinungen war variabel, die Symptome traten innerhalb weniger Tage auf und dauerten sowohl bei abruptem Absetzen als auch bei Ausschleichen des entsprechenden Medikaments wenige Wochen. Paroxetin wurde hierbei am häufigsten und stärksten mit dem Vorliegen von Absetzerscheinungen in Verbindung gebracht. Häufig wurden Absetzphänome als drohendes Rezidiv der Ursprungserkrankung missinterpretiert.

Schlussfolgerungen: Die Wirkstoffklasse der SSRI muss in der klinischen Praxis stärker mit dem möglichen Auftreten von Absetzerscheinungen in Verbindung gebracht werden.

\section{- Kommentar von Julia Becker-Sadzio, Tübingen}

\section{Empfehlung zum Absetzen von SSRI ändern?}

Bei der vorliegenden Arbeit handelt es sich um ein systematisches Review zur Untersuchung von Absetzerscheinungen bei Unterbrechungen in der Behandlung von psychischen Erkrankungen mit SSRI. Aufgrund der hohen Heterogenität der einbezogenen Studien erfolgte keine Metaanalyse der Daten. Neben randomisierten kontrollierten Studien wurden auch Studien mit deutlich geringerem Evidenzgrad einbezogen. Im Hinblick auf die jeweils mit SSRI behandelten psychischen Erkrankungen, Art und Dauer der Behandlungen sowie der Erhebung der entsprechend aufgetretenen Absetzerscheinungen bestand ebenfalls keine Einheitlichkeit. Dies sollte bei der

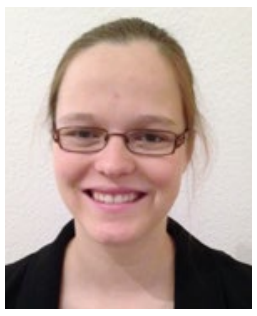

Julia Becker-Sadzio, Tübingen

Klinik für Psychiatrie und Psychotherapie Universitätsklinikum Tübingen

E-Mail: Julia.Sadzio@med.uni-tuebingen.de
Einschätzung der Ergebnisse kritisch bedacht werden. Für die tägliche Praxis sind die Ergebnisse vor allem dahingehend von Relevanz, als dass ein stufenweises Ausschleichen eines SSRI im Vergleich zu abruptem Absetzen das Auftreten von Absetzerscheinungen nicht verhindern oder deren Verlauf verkürzen kann, sodass die Empfehlungen zur Beendigung der Therapie mit SSRI gegebenenfalls modifiziert werden können. Die Kenntnis, dass Paroxetin scheinbar am häufigsten und stärksten mit Absetzerscheinungen in Verbindung gebracht wird, sollte vor allem in einer entsprechenden Aufklärung betroffener Patienten berücksichtigt werden.

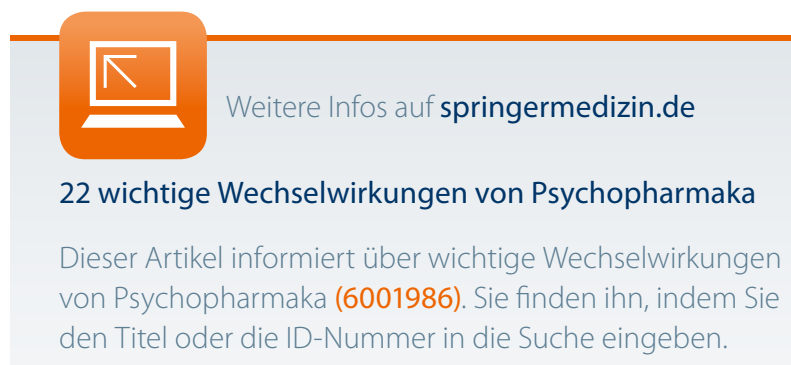

